\title{
A Japanese Semantic Network built on a Pulsed Neural Network with encoding Associative Concept Dictionaries
}

\author{
Takuya Sakaguchi \\ Media and Governance \\ Keio University \\ sakataku@sfc.keio.ac.jp
}

\author{
Shun Ishizaki \\ Environmental Information \\ Keio University \\ ishizaki@sfc.keio.ac.jp
}

\begin{abstract}
A semantic network with the dynamics such as spreading activity is available for various systems as well as the structure model of knowledge. In this study we suggest a practical dynamic semantic network available for NLP, which has the structure from associative concept dictionaries and the dynamics from a pulsed neural network. We built the semantic network by means of constructing the platform called "Brain Memory Model" based on a pulsed neural network first, then encoding data of associative concept dictionaries into it. We also constructed the module as one of the applications of the semantic network built on BMM to NLP, especially to simile understanding. The outputs of the module were understandable in general, indicating the possibility of our semantic network. We are now considering to expand the scale of a semantic network and to introduce the learning algorithm for its self-organization.
\end{abstract}

\section{Introduction}

A semantic network (Collins and Quillian, 1969) is a kind of network model, describing a structure of concepts preserved in a human brain. Basically it is formed with a number of concepts as nodes and relations between each concept as arks of a network. Several models of a semantic network have been suggested, some of them are dynamic models mentioning to the dynamics of a network such as spreading activity (Collins and Loftus, 1975). These models are available not only for structure models about concepts but also for some useful systems of associating, reasoning or natural language processing (NLP).

When considering to design such a dynamic and practical model of a semantic network, we have to mention to at least two problems, i.e. how to construct a large-scale structure and how to provide a dynamics of a network. In this study, we solved these problems by means of applying the database called "Associative Concept Dictionary" for its structure and the architecture constructed with a pulsed neural network for its dynamics, building a semantic network model and considered its availability for NLP, especially for simile understanding.

\section{Purpose}

As mentioned above, the purpose of this study is to build a semantic network with data of associative concept dictionaries and architecture of a pulsed neural network.

We would separate this study in three steps shown below. First we constructed the architecture based on a pulsed neural network, as the platform in which a semantic network is built. A pulsed neural network (Domany et al., 1994) is a kind of neural network model often designed with integrated and fire units, which is a more strict model to a real neuron in terms of considering a time course of a membrane potential of each neuron as well as its firing. Since the membrane potential is not reset but retained for some time 
even after its increase in a pulsed neural network, it is possible to realize spatio-temporal integration of firings. We constructed the platform for a semantic network with this neural network model, which is named "Brain Memory Model" or simply "BMM".

As the second step of this study we built a semantic network on BMM, by means of encoding the data of associative concept dictionaries into it. An associative concept dictionary (Okamoto and Ishizaki, 1998) is a large-scale database about concepts and their relations, gathered through cognitive experiments where a human subject answers some concepts associated from a stimulus concept along a certain type of relation. Each associated concept is registered to the database with its type of relation, values of his/her reaction time, reaction order and reaction rate and the distance to the stimulus concept is calculated from these values. Here we show a part of data of an associative concept dictionary (See Figure 1).

\begin{tabular}{|l|l|l|r|r|r|r|}
\hline Stimulus Word & Relation & Associative Word & Ans. Time & Ans. Order & Ans. Ratio & Distance \\
\hline Snow & ISA & Crystal & 0.30 & 1.00 & 0.10 & 6.49 \\
\hline Snow & ISA & Weather & 0.35 & 1.00 & 0.20 & 3.41 \\
\hline Snow & ISA & Water & 0.29 & 1.00 & 0.20 & 3.41 \\
\hline Snow & ISA & Nature & 0.13 & 1.00 & 0.20 & 3.41 \\
\hline Snow & ISA & Object & 0.34 & 1.33 & 0.30 & 2.49 \\
\hline Snow & HAS & Avalanche & 0.87 & 3.00 & 0.10 & 7.15 \\
\hline Snow & HAS & Hail & 0.43 & 2.00 & 0.10 & 6.82 \\
\hline Snow & HAS & Snowstorm & 0.68 & 2.50 & 0.20 & 3.91 \\
\hline Snow & HAS & Hailstone & 0.48 & 2.50 & 0.20 & 3.91 \\
\hline Snow & HAS & Sleet & 0.36 & 1.33 & 0.30 & 2.49 \\
\hline Snow & HAS & Powdery Snow & 0.47 & 1.75 & 0.40 & 2.12 \\
\hline Snow & CAN & Melt & 0.62 & 3.00 & 0.20 & 4.07 \\
\hline Snow & CAN & Fall & 0.35 & 1.50 & 1.00 & 1.11 \\
\hline$:$ & $:$ & $:$ & $:$ & $:$ & $:$ & $:$ \\
\hline
\end{tabular}

Figure 1. Data of Associative Concept Dictionary.

As a result of encoding these data into BMM, it is possible to build a semantic network with a rich structure about concepts and a dynamics of spatiotemporal integration.

Finally, we mentioned to the availability of this semantic network for NLP. In this study we constructed the system for simile understanding which transfers a typical simile expression "The $T$ seems just like $S^{\prime \prime}$ into a simile-understood expression "The $T$ is so $U$ ", with the help of behaviors of the semantic network built on BMM. Here we defined " $T$ " and " $S$ " in the simile expression as a simple noun concept named "target concept" and "source concept", while " $U$ " in the simile-understood expression as "goal concept" which is output by the system.

\section{Methods}

\subsection{Constructing Brain Memory Model}

The first step of this study is to construct Brain Memory Model as the platform to build a semantic network. We designed BMM as shown below, which is composed with several modules (See Figure 2). In this section we would explain the architecture of BMM with mentioning to the roles of these modules.

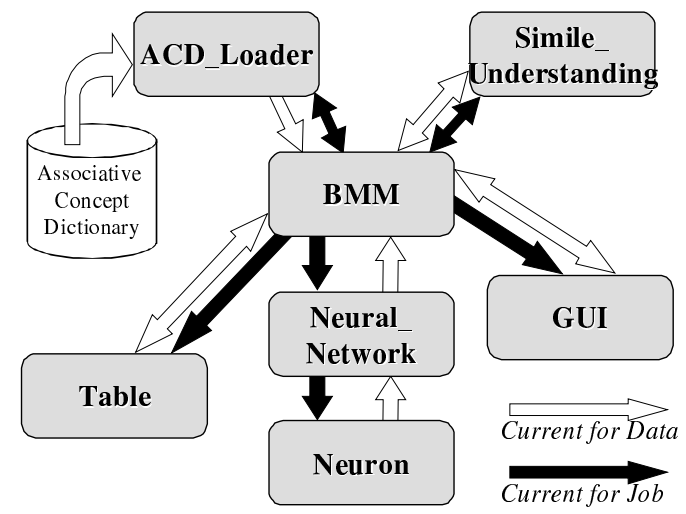

Figure 2. Module Composition of BMM.

Neural_Network module is one of the most important modules, which is the base of BMM describing its structure and dynamics. Note that Neuron module composing Neural_Network module is designed as integrated and fire unit, thus Neural_Network module is regarded as a pulsed neural network model. Neural_Network module is designed as a multiple layered structure, and each layer is possible to treat in a separate manner. It is able to set the parameter such as a firing rule of neurons, value of threshold and number of firing neurons for each layer. It is also possible to knock out any layer instantly, thus we could control the area of network activity in BMM.

Table module plays the role to preserve the information of concepts encoded in BMM. When starting BMM program, Table module is built with empty because there are no data encoded at this moment. Thus we have to consider encoding data of associative concept dictionaries into BMM, where ACD_Loader module plays the important role. This procedure would be explained in the next section again.

Simile_Understanding module is the application of BMM rather than BMM itself, which transfers a simile expression into a simile-understood expression with the help of the semantic network 
built on BMM. The detail of this module would be explained in section 3.3.

GUI module provides the interface of BMM. It accepts various kinds of inputs about activities or parameters, displaying the current of status or condition in BMM on the other hand (See Figure $3)$.

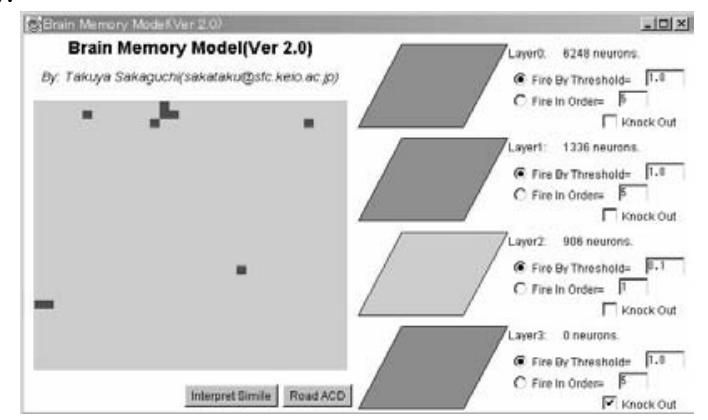

Figure 3. GUI Image of BMM.

Finally, the center $B M M$ module controls and organizes all other modules as a main module.

\subsection{Building a Semantic Network}

In this section we would mention to the second step of the study, i.e. the method to build a semantic network with encoding associative concept dictionaries into BMM architecture.

Encoding Concept and Distance: The core idea of encoding is to assign one concept of associative concept dictionaries into one neuron of BMM, while the distance value between any two concepts into the synapse weight between neurons indicating those of two concepts (See Figure 4).

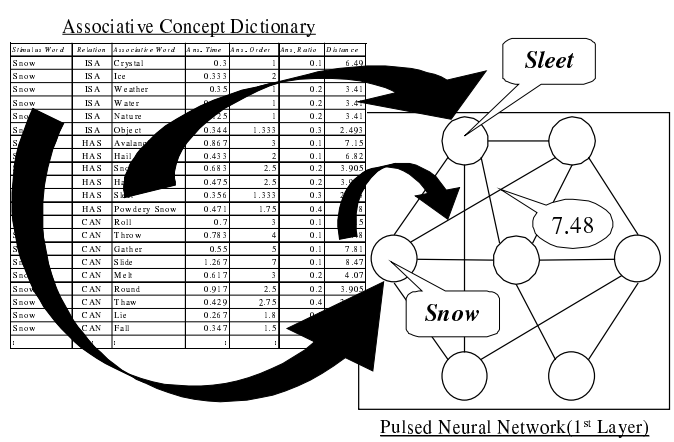

Figure 4. Encoding Data of Associative Concept Dictionaries into BMM.

For instance, if the data about concept "snow", concept "sleet" and the distance value between two of them are loaded from the associative concept dictionary into BMM, then two neurons labeled "snow" and "sleet" are prepared in BMM and connected them with each other by a synapse with a certain weight calculated from the distance value. In this way, concepts are expressed as neurons, while relations are expressed as synapses in BMM.

This procedure of encoding is achieved by $A C D \_L o a d e r$ module and Table module. In detail, when $A C D \_L o a d e r$ module read one datum of a concept from an associative concept dictionary, first it searches whether the neuron assigned that concept already exists in BMM, and if not, prepares a new neuron to assign it. Secondly the information of the assigned neuron number and the concept name is paired and preserved in Table module.

On the other hand, when the datum about distance is read, ACD_Loader module also searches and prepares the synapse of which the relation is consistent, then calculates the appropriate weight of the synapse from the distance value. The equation for the calculation is described as

$$
w_{i j}=k_{1} /\left(d_{i j}+k_{2}\right)
$$

where $w_{i j}$ is a synapse weight from neuron $j$ to neuron $i . d_{i j}$ is a distance between concept $j$ and concept $i$ and $k_{1}, k_{2}$ are both constant values adjusting the order of synapse weights. The values of synapse weights are preserved not as data in Table module but as parameters in Neural_Network module.

Encoding Type of Relation: We should consider not only the distance of relation but also the type of relation to encode. As mentioned above, the data of associative concept dictionaries are gathered through cognitive experiments, where the type of relation for associated concepts to answer has been regulated. In this way, there are several types of relation registered in associative concept dictionaries, e.g. generalized, specific, material, attribute, action and so on (Okamoto and Ishizaki, 1998). The easiest way to encode them is to attach a relation tag to each synapse in BMM, however, adding extra information in the architecture might be cause to make the structure more complicated. Thus in this study, we applied a multiple layered structure of BMM to classify those data, encoding concepts belonged in another type of relation in another layer. For instance, we could treat stimulus concepts itself, associated 
concepts belonged in attribute and another type of associated concepts belonged in action in a separate manner, with preparing three layers to encode each of them. This way of encoding realizes not only a smart structure of BMM but also more comfortable analysis, such as a knock out simulation of each relation.

Outline of the Semantic Network: As a result of encoding explained above, a semantic network was built with the structure based on associative concept dictionaries and the architecture based on a pulsed neural network of BMM. This semantic network consists of multiple layers, and concepts in each layer have intra- and inter-layer connections with other concepts (See Figure 5).

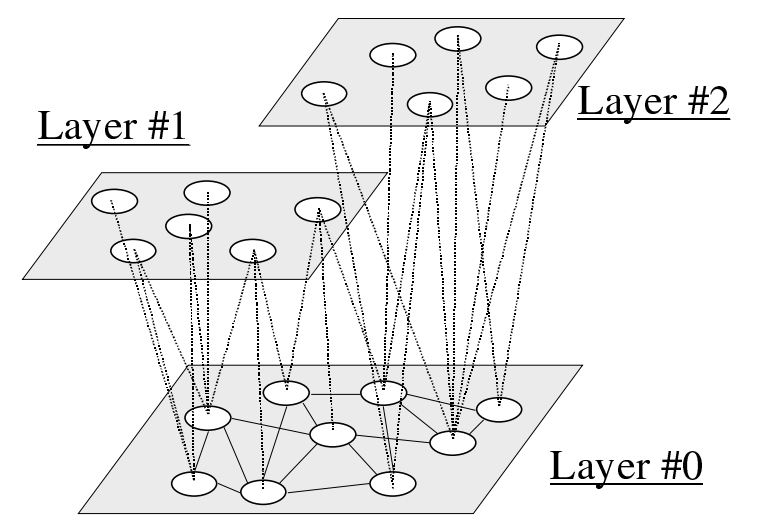

Figure 5. Multiple Layer Structure of Semantic Network.

We could regard a firing of the neuron as a recalling of the concept assigned by that neuron. Moreover, since the dynamics of BMM that a firing of a neuron spreads to other neurons via synapses is available, it is possible to associate relative concepts triggered by a recalling of one concept.

\subsection{Simile Understanding}

Simile_Understanding module introduced in section 3.1 is the independent module from BMM itself, designed as one of the applications of the semantic network built on BMM. The purpose of this module is to understand simile expressions, with transferring a typical simile expression "The $T$ seems just like $S^{\prime \prime}$ into a simile-understood expression "The $T$ is so $U$ " with the help of the semantic network on BMM. In this study we treated only this syntax as a simile expression and defined $T$ and $S$ are simple noun concepts, in order to avoid mentioning to morphological analysis.

Procedure of Simile Understanding: There are several hypotheses suggested for simile understanding. Comparison theory (Ortony, 1979) is one of them, which achieves simile understanding by means of finding a common concept between vehicle and topic. We followed this hypothesis and considered the problem just as a searching problem of goal concept $U$ from target concept $T$ and source concept $S$, where $U$ is considered as the common concept connected with both of $T$ and $S$.

Simile_Understanding module solves this searching problem on the semantic network with the help of the dynamics to associate relative concepts, which has been explained above. In detail, the current of the procedure could be divided by 7 processes as shown below:

(1) Recognize the simile expression input

(2) Search and stimulate target concept in the semantic network

(3) The stimulus spreads to other concepts connected with the target concept

(4) Search and stimulate source concept in the semantic network

(5) The stimulus spreads to other concepts connected with the source concept

(6) Two stimuli are integrated only in the concept connected with both of target and source concept and make it fire finally

(7) Output the concept fired as goal concept in the simile-understood expression

Here we would trace the current of these processes considering the case of a typical simile expression "The skin seems just like snow". When a user input target concept "skin" and source concept "snow" into the system, first it searches the neuron indicating "snow" in BMM and stimulates it strongly enough for its firing. At the next step, the firing of the neuron spreads in a network and stimulates other neurons connected with it, in this case the neuron indicating "white" and the neuron indicating "cold" are stimulated. On the other hand, the system also fires the neuron of source concept "skin", causing the trigger of spreading activity to stimulate other connected neurons, indicating such as "white", "beautiful" or "smooth". Note that the neuron of "white", which is the only 
neuron connected with both of "snow" and "skin", is stimulated by two neurons to increase its membrane potential in two times. As a result, the most excited neuron in BMM would be the one indicating "white" and the system finally outputs "white" as the primary goal concept.

Asymmetry of Target Concept and Source Concept: In general, it is not a good idea to treat target concept and source concept equally, since the influence of those of two concepts for the simile understanding are not equivalent. We could realize this easily, considering that the simile expression "The person seems just like a robot" and the other expression "The robot seems just like a person" give us quite different impressions, though the differences between those of two expressions are nothing but only target concept and source concept have been exchanged.

Kusumi investigated about this problem, and concluded that the influence of source concept to simile understanding is about twice as strong as that of target concept (Kusumi, 1987). In this study we followed this investigation and differentiated the influence of target concept and source concept, by means of putting a short time delay between the process of stimulating for target concept (process (2)) and that for source concept (process (4)). The dynamics of the semantic network is given by BMM based on a pulsed neural network model, where the membrane potential of a neuron decays as time advances and a stimulus in past becomes less and less influential. We applied this aspect to make the later stimulus of source concept more influential than the earlier stimulus of target concept, putting 2 to 3 time steps of delay between the process (2) and (4) that keeps the best equilibrium of ratio $2: 1$ for simile understanding.

\section{Result}

\subsection{Network Constructing}

In order to build a semantic network on BMM, first we considered to adopt two kinds of associative concept dictionaries to load, i.e. $A C D 50$ and $A C D 10$. $A C D 50$ is the associative concept dictionary constructed through cognitive experiments with 50 human subjects for each concept, containing about 60,000 entries with a certain level of reliability. On the other hand, $A C D 10$ is constructed with 10 human subjects, containing more than 70,000 entries but with less reliability. Both of associative concept dictionaries consist of only Japanese concepts. Loading these multiple databases realized to build a richer network with wide and deep knowledge on BMM. Secondly, we considered data with answering rate less than $20 \%$ for $A C D 10$ and $4 \%$ for $A C D 50$ as junk data and ignored them when loading data into BMM. And finally we prepared three layers in BMM and separated concepts by their types of relation with loading them in other layers, i.e. Layer \#0 for stimulus concepts and associated concepts belonged in generalized or specific, Layer \#1 for associated concepts in action and Layer \#2 for associated concepts in attribute. In associative concept dictionaries, almost all of associated concepts belonged in action are verb and concepts in attribute are adjective, thus this proceeding has the purpose to separate concepts by their parts of speech.

The table shown below donates the properties of the semantic network built on BMM, as a result of loading two kinds of associative concept dictionaries with the configurations explained above (See Table 1).

\begin{tabular}{lr}
\hline & Num of Concerts \\
\hline Layer $\# 0$ & 6248 \\
Layer \#1 & 1336 \\
Layer \#2 & 906 \\
Total & 8490
\end{tabular}

Table 1. Number of Concepts in Semantic Network.

\subsection{Simile Understanding}

In this section, we would display the outputs of Simile_Understanding module, which transfers a simile expression input into a simile-understood expression output.

First we selected several examples of simile expressions for the demonstration of Simile_Understanding module. Since it searches goal concept with stimulating target concept and source concept in the semantic network, the two of concepts in a simile expression must be registered in the semantic network first. Additionally, if there is no relation between those concepts at all, the module might not output the appropriate goal concept. Taking into account these points enough, we finally selected 10 examples which are displayed in the table below (See Table 2). The table also displays the output of the module for 
each input. For instance, we could understand from this table that the simile expression "The skin seems just like snow" was input at Task \#1 and the simile-understood expression "The skin is so white" was output by the module.

\begin{tabular}{l|ccc}
\hline Task No. & T & S & U \\
\hline Task \#1 & Skin & Snow & white \\
Task \#2 & Mind & Snow & cold \\
Task \#3 & Mind & Flame & hot \\
Task \#4 & Robot & Person & wise \\
Task \#5 & Person & Robot & cold \\
Task \#6 & personality & Stone & hard \\
Task \#7 & Thought & Sky & deep \\
Task \#8 & Dream & Earth & huge \\
Task \#9 & Word & Needle & sharp \\
Task \#10 & Life & Violet & flail \\
\hline
\end{tabular}

Table 2. Input/Output for Simile Understanding.

From the results of Task \#1, Task \#2 and Task \#3, we could find that the module certainly referred to both of source concept and target concept for simile understanding. On the other hand, we could also find from the results of Task \#4 and \#5 that the asymmetry of two concepts was considered appropriately in the procedure. And from the whole results, it might be possible to say that the module could output a simile-understood expression understandable in general for each simile expression, as long as target concept and source concept are registered and related in the semantic network.

We finally concluded from these considerations that the semantic network built on BMM with encoding associative concept dictionaries has the probability for the application to NLP such as simile understanding.

For more evaluation of Simile_Understanding module, we could refer the study by Sakaguchi and Ishizaki where the difference between outputs of the module and answers by human beings has been investigated, concluding that the module could follow human beings in most simile expressions (Sakaguchi and Ishizaki, 2000).

\section{Conclusion}

In this study, first we constructed BMM architecture, which would be the platform in which a semantic network was built. It was designed based on a pulsed neural network, where a spatiotemporal integration of firings is possible. Secondly we considered the way of encoding data of associative concept dictionaries into architecture of BMM, i.e. concepts into neurons, distance values into synapse weights and types of relation into layers of BMM. We indeed encoded two kinds of associative concept dictionaries into BMM and built a semantic network containing totally about 8,500 concepts. Finally, we constructed the module for simile understanding, which transfers a typical simile expression into an appropriate simile-understood expression by means of searching a common concept connected both of target concept and source concept in the semantic network built on BMM.

There might be some problems remained for future works. The first problem is that the number of concepts composing a semantic network is less than 10,000 , which is not enough for a practical semantic network. We should encode more data of associative concept dictionaries, or consider to adopt another database such as WordNet (Miller et al., 1993). If we succeed to expand the scale of a semantic network with more concepts, it is expected that much more simile expressions become available for the evaluation of Simile_Understanding module.

The second problem we consider is that synaptic weights are treated as constant value in this study. We would like to improve this point with introducing the learning algorithm for BMM. It must make the semantic network much more dynamic model, which is possible of learning, selforganizing and optimizing its structure.

Finally, we would like to keep improving BMM toward the complete model, explaining the whole memory structure in the brain and realizing any simulation of intellectual activity, that would be called the "real" Brain Memory Model in the future.

\section{References}

Collins,A.M. and Loftus,E.F., "A Spreading-Activation Theory of Semantic Processing", In Psychological Review, Vol.82-6, pp.407-428, 1975.

Collins,A.M. and Quillian,M.R., "Retrieval Time from Semantic Memory", In Journal of Verbal Learning and Verbal Behavior, Vol.8, pp.240-247, 1969.

E.Domany, J.L.van Hemmen, K.Schulten, "Models of Neural Networks II --- Temporal Aspects of Coding 
and Information Processing in Biological Systems", Springer-Verlag, 1994.

Kusumi,T., "Effects of categorical dissimilarity and affective similarity between constituent words on metaphor appreciation", Journal of Psycholinguistic Research, 16, pp.577-595.

Miller,G.A., Beckwin,R., Fellbaum,C., Gross,D., Miller,K. and Tengi,R., "Five Papers on WordNet", Cs1 report 43, Cognitive science Laboratory Princeton University, 1993.

Okamoto,J. and Ishizaki,S., "Construction of Electronic Concept Dictionary and Quantification of Concept Space", SIG-NL-130 IPSJ, 1998.

Ortony,A., "Beyond literal similarity", Psychological Review, 86, pp.161-280, 1979.

Sakaguchi,T. and Ishizaki,S., "Simile Understanding System Using Associative Concept Dictionary and Pulsed Neural Network", Pacific Association for Computational Linguistics, 2000. 